\title{
Knockdown of stromal interaction molecule 1 inhibits proliferation of colorectal cancer cells by inducing apoptosis
}

\author{
DONG YANG, XIAOYU DAI, KEQIANG LI, YANGYANG XIE, JIANPEI ZHAO, \\ MINGJUN DONG, HUA YU and ZHENFANG KONG
}

\author{
Department of Surgery, The Second Hospital of Ningbo, Ningbo, Zhejiang 315000, P.R. China
}

Received September 23, 2016; Accepted August 8, 2017

DOI: $10.3892 / \mathrm{ol} .2018 .8437$

\begin{abstract}
Stromal interaction molecule 1 (STIM1) is an endoplasmic reticulum $\mathrm{Ca}^{2+}$ sensor which has been reported to be overexpressed in numerous types of cancer, and is involved in the cell proliferation, invasion, migration and metastasis frequently observed in cancer. However, the role of STIM1 in colorectal cancer (CRC) remains unknown. The purpose of the present study was to investigate the effect of STIM1 in human CRC. The expression of STIM1 was specifically knocked down using lentivirus-mediated small hairpin RNA (shRNA) interference techniques in the CRC cell lines HCT116 and SW1116. Subsequently, the efficiency of infection was confirmed using green fluorescent protein (GFP)-positive signals. The knockdown efficiency was further determined using the reverse transcription-quantitative polymerase chain reaction and western blotting analysis. As a result, CRC cell lines with STIM1 silenced were successfully constructed and subsequently employed in a series of cell function assays. Knockdown of STIM1 significantly suppressed cell proliferation and colony formation, as revealed by an MTT and colony formation assay. Furthermore, it was identified that STIM1 silencing may promote cell apoptosis through the induction of mitochondria-associated apoptosis, as was identified by increased expression levels of B-cell lymphoma 2 (Bcl-2)-associated death promoter, Bcl-2-associated X protein and poly(ADP-ribose) polymerase cleavage. Therefore, STIM1 may serve a critical role in the progression of CRC by regulating cell proliferation and apoptosis, which may provide a potential therapeutic target for the treatment of CRC.
\end{abstract}

Correspondence to: Mr. Keqiang Li or Mr. Xiaoyu Dai, Department of Surgery, The Second Hospital of Ningbo, 41 North West Street, Ningbo, Zhejiang 315000, P.R. China

E-mail: chasejxmc@163.com

E-mail: xiaoyu_dai@126.com

Key words: colorectal cancer, stromal interaction molecule 1, cell proliferation, apoptosis

\section{Introduction}

Colorectal cancer (CRC) is the third most commonly diagnosed malignancy in males and the second in females, accounting for $\sim 0.6$ million mortalities annually $(1,2)$. The development and progression of colorectal cancer is associated with multiple factors including lifestyle, environmental factors, and genetic and epigenetic changes $(3,4)$. Despite investigations into the etiology of CRC, the underlying molecular mechanisms of the disease remain incompletely understood.

Stromal interaction molecule 1 (STIM1) has previously been identified as a novel human gene, located in the imprinted gene domain of $11 \mathrm{p} 15.5$ which is also reported to be an important tumor suppressing gene region (5). $\mathrm{Ca}^{2+}$ is an almost ubiquitous intracellular messenger regulating a variety of physiological and pathological processes (6). Store-operated $\mathrm{Ca}^{2+}$ channels provide the major $\mathrm{Ca}^{2+}$ entry pathway in non-excitable cells, particularly in cancer cells. It has been demonstrated that STIM1 is an endoplasmic reticulum (ER) $\mathrm{Ca}^{2+}$ sensor and controls store-operated $\mathrm{Ca}^{2+}$ entry (SOCE) with ORAI1 (7). Once $\mathrm{Ca}^{2+}$ is released from the ER, STIMs are able to aggregate and translocate to the ER-plasma membrane to activate Orai1 (8). STIM1 has also been implicated in the proliferation, migration, invasion and metastasis in a number of types of cancer, including cervical cancer, non-small lung cancer, breast cancer and melanoma (9-12). Furthermore, STIM1 is a potential molecular marker for the diagnosis and progression of cancer $(13,14)$. However, there remain conflicting results about the functions of STIM1 in different cancer studies. Upregulation of STIM1-ORAI1-mediated SOCE appears to promote metastasis by stimulating the migration of breast tumor cells (15). Downregulation of STIM1-ORAI1-mediated SOCE was demonstrated to contribute to the increased resistance to apoptosis in prostate cancer cells (16). The molecular mechanisms underlying the regulation of signaling pathways by STIM1 in cancer require further investigation. Previous studies have demonstrated that STIM1 is associated with tumor metastasis in CRC $(17,18)$. However, the importance of the role served by STIM1 in cell growth and proliferation of CRC remains unclear.

In the present study, small hairpin RNA (shRNA) silencing of STIM1 in CRC cell lines was performed and it was revealed that STIM1 knockdown was able to inhibit proliferation and induce apoptosis of CRC cells. Several pro-apoptotic markers 
were also studied to reveal the molecular mechanism underlying STIM1 silencing-promoted cell apoptosis in CRC. The results of the present study suggest that STIM1 may be essential for the proliferation and tumorigenesis of CRC.

\section{Materials and methods}

Cell lines and culture. Human CRC cell lines SW1116 and HCT116, and 293T cells were obtained from the Cell Bank of the Chinese Academy of Sciences (Shanghai, China). HCT116 cells were cultured in RPMI-1640 medium (GE Healthcare, Chicago, IL, USA) with $10 \%$ fetal bovine serum (FBS; Biowest, MO, USA). SW1116 cells and 293T cells were cultured in Dulbecco's modified Eagle's medium (GE Healthcare) supplemented with $10 \% \mathrm{FBS}$ at $37^{\circ} \mathrm{C}$ in a humidified incubator containing $5 \% \mathrm{CO}_{2}$.

Lentiviral packaging and cell infection. An shRNA targeting human STIM1 (shSTIM1; NM_003156.3) was designed using online shRNA tools (Invitrogen; Thermo Fisher Scientific, Inc., Waltham, MA, USA): shRNA (5'-CTAGCG CCTGGATGATGTAGATCATAATTCAAGAGATTATGA TCTACATCATCCAGGTTTTTT-3'). The non-silencing shRNA (shCon) sequence (5'-TTCTCCGAACGTGTC ACGTCTCGAGACGTGACACGTTCGGAGAATTTTT-3') was used as a negative control. All oligonucleotides were synthesized and cloned into NheI/PacI-linearized pFH-L vector (Shanghai Hollybio Co., Ltd., Shanghai, China), which contains green fluorescent protein (GFP) as a reporter marker. The lentiviruses were subsequently generated by co-transfecting 293T cells with the shSTIM1 plasmid and pVSVG-I and pCMV-dR8.92 helper (Shanghai Hollybio Co., Ltd.). Lentiviruses were purified using ultracentrifugation with centrifugal force of $80,000 \mathrm{xg}$ at $4^{\circ} \mathrm{C}$ for $60 \mathrm{~min}$. To determine the viral titer, $293 \mathrm{~T}$ cells were transfected with lentiviruses and following this, green fluorescent cells under fluorescence microscopy were observed and the viral titer was estimated. For cell infection, HCT116 $\left(1 \times 10^{5}\right.$ cells/well) and SW1116 cells $\left(4 \times 10^{4}\right.$ cells/well) were plated in 6-well plates and then infected with lentiviruses (shCon and shSTIM1) at a multiplicity of infection of 60 and 50, respectively, for $96 \mathrm{~h}$. GFP-positive signals were observed using fluorescence microscopy to evaluate infection efficiency.

Reverse transcription-quantitative polymerase chain reaction (RT-qPCR) analysis. Total RNA was extracted using TRIzol reagent (Invitrogen; Thermo Fisher Scientific, Inc.) according to the manufacturer's protocol. RNA (1 $\mu \mathrm{g})$ was reverse-transcribed into cDNA using a Takara Reverse Transcription system (Takara Biotechnology Co., Ltd., Dalian, China). RT-qPCR was conducted on a BioRad Connect RT-PCR platform using CFX Manager software and the Precision Melt Analysis system (version 1.0; Bio-Rad Laboratories, Inc., Hercules, CA, USA) using a volume of $20 \mu \mathrm{l}(10 \mu \mathrm{l} 2 \mathrm{X}$ SYBR Premix Ex Taq, $0.5 \mu 1$ forward and reverse primers, $5 \mu \mathrm{l}$ cDNA and $4.5 \mu 1$ double-distilled water) under the following conditions: Initial denaturation at $95^{\circ} \mathrm{C}$ for $1 \mathrm{~min}$ and 40 cycles of denaturation at $95^{\circ} \mathrm{C}$ for $5 \mathrm{sec}$ and annealing and extension at $60^{\circ} \mathrm{C}$ for $20 \mathrm{sec}$. The relative expression levels of STIM1 mRNA were calculated using the $2^{-\Delta \Delta \mathrm{Cq}}$ method (19), and $\beta$-actin was used as the reference gene. The primers used were as follows: STIM1, 5'-AGCCTCAGCCATAGTCACAG-3' (forward) and 5'-TTCCACATCCACATCACCATTG-3' (reverse); B-cell lymphoma 2 (Bcl-2)-associated death promoter (Bad), 5'-GGC ACAGCAACGCAGATG-3' (forward) and 5'-CGGGTGGAG TTTCGGGATG-3' (reverse); Bcl-2-associated X protein (Bax), 5'-TGGGCTGGACATTGGACTTC-3' (forward) and 5'-AGCACTCCCGCCACAAAG-3' (reverse); and $\beta$-actin, 5'-GTGGACATCCGCAAAGAC-3' (forward) and 5'-AAA GGGTGTAACGCAACTA-3' (reverse).

Western blotanalysis. Total protein was extracted using 2X SDS Lysis Buffer (100 mM Tris, 4\% SDS, 10\% glycerol, $200 \mathrm{mM}$ $\mathrm{NaCl}, 2 \mathrm{mM}$ EDTA, pH=6.8) from SW1116 and HCT116 cells, and then $30 \mathrm{~g}$ protein was separated by SDS-PAGE with $10 \%$ acrylamide and transferred onto polyvinylidene fluoride membranes. The membranes were probed with specific antibodies against STIM1 (cat. no. sc-166840; 1:1,000 for HCT116 cells and 1:500 for SW1116 cells; Santa Cruz Biotechnology, Inc., Dallas, TX, USA), poly(ADP-ribose) polymerase (PARP; cat. no. 9542; 1:1,000; Cell Signaling Technology Europe, B.V., Leiden, The Netherlands) or GAPDH (cat. no. 10494-1-AP; 1:50,000 for HCT116 cells and 1:10,000 for SW1116 cells; Proteintech Group Inc., Chicago, IL, USA) at $4^{\circ} \mathrm{C}$ overnight. Membranes were subsequently incubated with horseradish peroxidase-conjugated goat anti-mouse (cat. no. sc-2005; 1:5,000; Santa Cruz Biotechnology, Inc.) or goat anti-rabbit (cat. no. sc-2054, 1:5,000; Santa Cruz Biotechnology, Inc.) immunoglobulin $\mathrm{G}$ antibody and visualized using the Pierce enhanced chemiluminescence kit (Thermo Fisher Scientific, Inc.), according to the manufacturer's protocol.

MTT assay. Cell proliferation was determined using an MTT assay (Beyotime Institute of Biotechnology, Haimen, China), according to the manufacturer's protocol. Briefly, following lentivirus infection for $120 \mathrm{~h}$, HCT116 cells and SW1116 cells were seeded into 96 -well plates at a density of $2 \times 10^{3}$ or $2.5 \times 10^{3}$ cells/well, respectively. At the indicated time points $(1,2,3,4$ and 5 days), MTT solution $(5 \mathrm{mg} / \mathrm{ml})$ was added to each well prior to incubation at $37^{\circ} \mathrm{C}$ in a humidified chamber for $4 \mathrm{~h}$. Acidic isopropanol (10\% SDS, 5\% isopropanol and $0.01 \mathrm{M} \mathrm{HCl}$ ) was added to stop the reaction. After $10 \mathrm{~min}$ of incubation, the absorbance was measured using a microplate reader at a wavelength of $595 \mathrm{~nm}$.

Colony formation. Following lentivirus infection for $120 \mathrm{~h}$, SW1116 cells in the exponential growth phase were plated into 6 -well plates at 500 cells/well. Following continuous culture for 10 days, the RPMI-1640 medium was then discarded. The cells were washed with PBS ( $\mathrm{pH} 7$ ) twice, fixed with methyl alcohol for $20 \mathrm{~min}$, stained with crystal violet for $20 \mathrm{~min}$, washed with distilled water and dried. Colonies were counted by eye.

Cell apoptosis analysis. Apoptosis was evaluated using the Annexin V/7-aminoactinomycin-D (7-AAD) double staining assay (eBioscience; Thermo Fisher Scientific, Inc.). Briefly, SW1116 cells were reseeded into a 6-well plate at $2 \times 10^{5}$ cells/well 5 days after lentiviral infection. When cell 
A
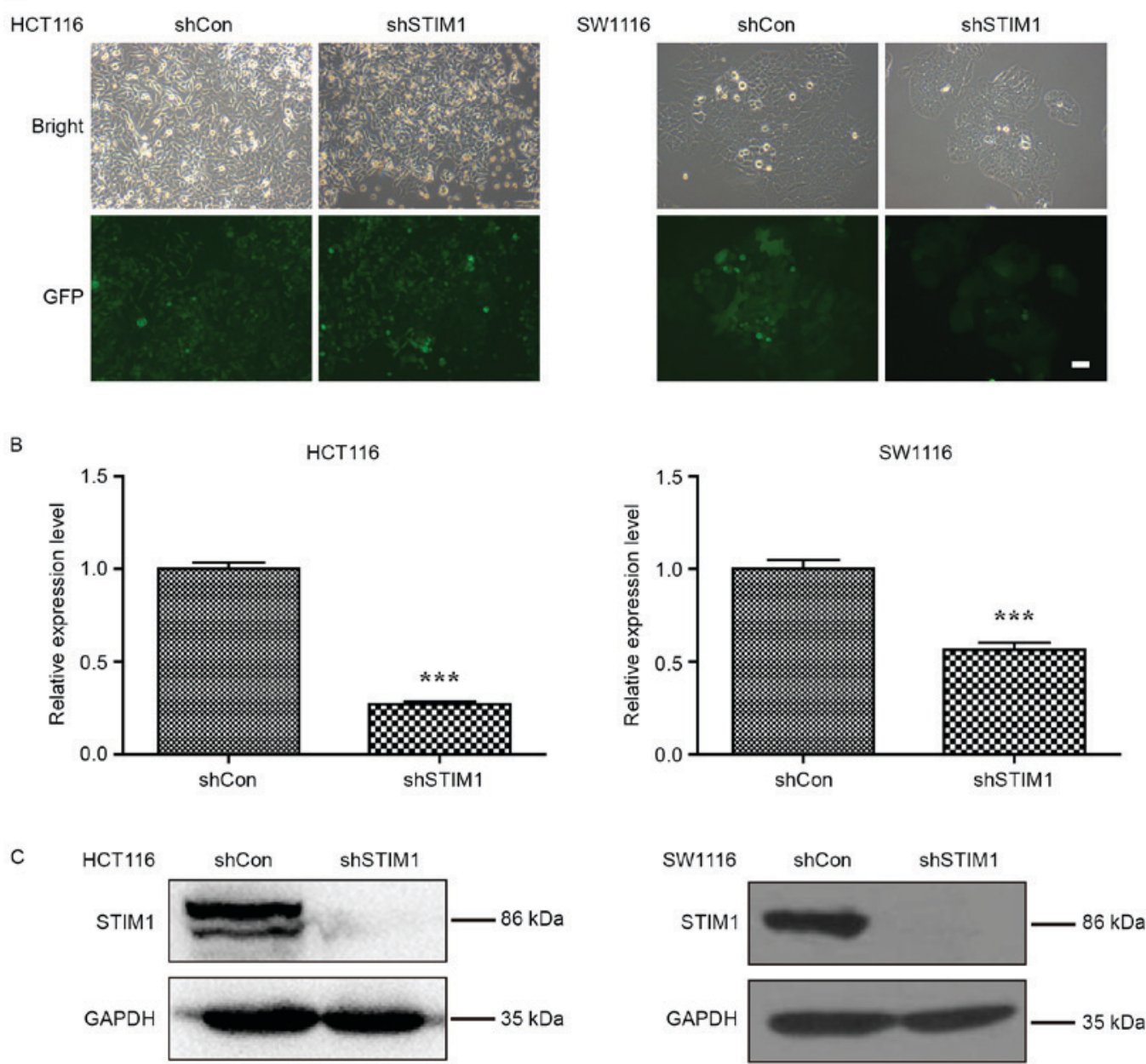

Figure 1. Knockdown of STIM1 expression in CRC cell lines HCT116 and SW1116 by shSTIM1. (A) Representative images of green fluorescent protein expression captured in HCT116 and SW1116 cells using a fluorescence microscope. (B) Reverse transcription-quantitative polymerase chain reaction analysis of STIM1 mRNA levels n HCT116 and SW1116 cells following shSTIM1 infection. Results are expressed as the mean \pm standard deviation from three independent replicate experiments. ${ }^{* * *} \mathrm{P}<0.001$ vs. shCon. (C) Western blot analysis of STIM1 protein levels in HCT116 and SW1116 cells following shSTIM1 infection. STIM1, stromal interaction molecule 1; shSTIM1, shRNA targeting human STIM1; shCon, non-silencing shRNA; CRC, colorectal cancer.

confluence reached $80 \%$, cells were collected, washed twice with PBS at $4^{\circ} \mathrm{C}$ and resuspended in binding buffer. Finally, cells were stained with Annexin V and 7-AAD solution, and the apoptotic cells were analyzed using flow cytometry.

Statistical analysis. Data analyses were performed using GraphPad Prism software (GraphPad Software 5.0, Inc., La Jolla, CA, USA). All results are expressed as the mean \pm standard deviation from three independent replicate experiments. $\mathrm{P}<0.05$ was considered to indicate a statistically significant difference, using one-way analysis of variance followed by Student's t-test.

\section{Results}

Lentivirus-mediated shRNA efficiently suppresses STIMI expression in CRC cells. To study the biological role of STIM1 in CRC, the expression of STIM1 was specifically knocked down in CRC cell lines, HCT116 and SW1116. As presented in Fig. 1A, the majority of HCT116 and SW1116 cells expressed GFP-positive signals, suggesting satisfactory infection efficiency. Furthermore, the knockdown efficiency of STIM1 was investigated in these two cell lines using RT-qPCR and western blot analysis. As presented in Fig. 1B, the mRNA levels of STIM1 were significantly decreased in HCT116 and SW1116 cells following STIM1 knockdown $(\mathrm{P}<0.001$ when compared with shCon). Consistent with qPCR results, the protein levels of STIM1 were decreased in the shSTIM1 group compared with the shCon group in HCT116 and SW1116 cells, respectively (Fig. 1C). These results suggest that the lentivirus-mediated shRNA was able to significantly suppress the endogenous STIM1 expression in HCT116 and SW1116 cells.

Knockdown of STIM1 significantly inhibits CRC cell proliferation and colony formation ability. Proliferation of shCon- or shSTIM1-transfected HCT116 and SW1116 cells was measured using an MTT assay daily for 5 days. The result demonstrated that STIM1-specific shRNA caused a significant decrease in the proliferation of HCT116 and SW1116 cells ( $\mathrm{P}<0.001$ vs. shCon; Fig. 2A). Notably, suppression of cell proliferation following knockdown of STIM1 was more evident in SW1116 cells than in HCT116 cells. As a result, SW1116 cells were selected to perform a colony formation assay. As presented in Fig. 2B and C, STIM1-depleted cells grew slowly and formed 

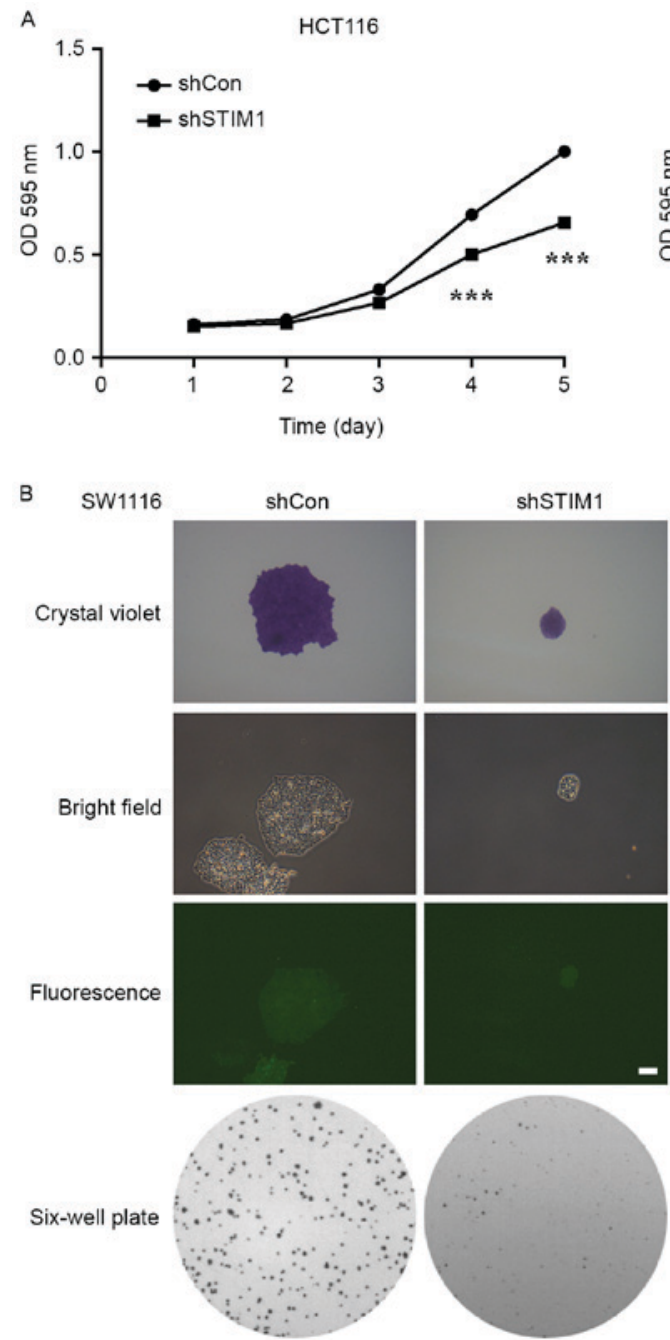

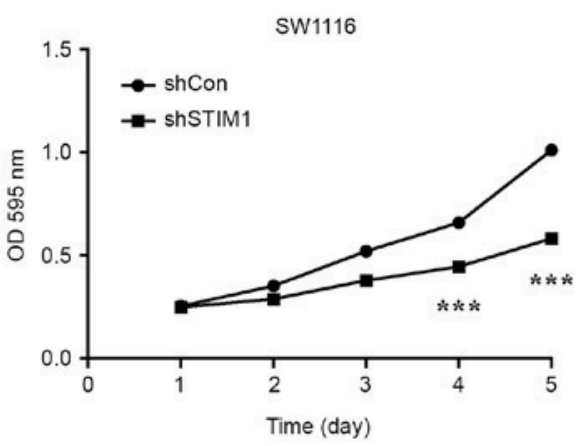

c

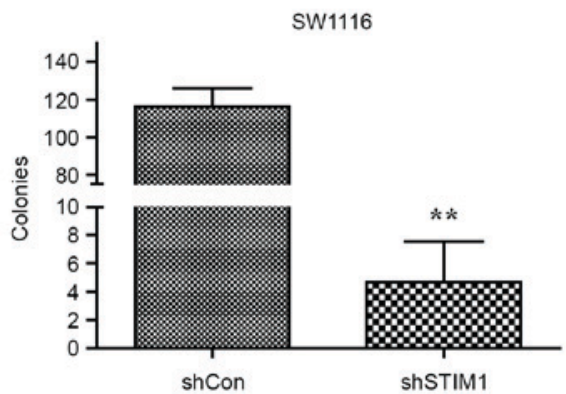

Figure 2. STIM1 silencing inhibits the viability and colony formation ability of CRC cells. (A) Knockdown of STIM1 significantly suppressed the viability of HCT116 and SW1116 cells, as determined using an MTT assay. Results are expressed as the mean \pm standard deviation from three independent replicate experiments. ${ }^{* * *} \mathrm{P}<0.001$ vs. shCon. (B) Representative images of colonies formed in SW1116 cells with two treatments (shCon and shSTIM1) measured using a colony formation assay. (C) Quantification of colony formation ability. The experiments were performed in triplicate and repeated in triplicate. ${ }^{* *} \mathrm{P}<0.01$ vs. shCon. STIM1, stromal interaction molecule 1; shSTIM1, shRNA targeting human STIM1; shCon, non-silencing shRNA; CRC, colorectal cancer; OD, optical density.

a few smaller colonies when compared with the control group during a 10-day period, which indicated that knockdown of STIM1 significantly impaired the colony formation ability of SW1116 cells $(\mathrm{P}<0.01)$. These results indicate that STIM1 silencing may inhibit the proliferation and colony formation ability of CRC cells in vitro.

STIM1 silencing promotes the apoptosis of CRC cells. Genes that are capable of inducing apoptosis are generally considered potential anticancer targets. To confirm whether STIM1 is a suitable potential molecular target for CRC, the Annexin V/7-AAD double staining apoptosis detection assay was used to investigate cell apoptosis in SW1116 cells following STIM1 knockdown. As presented in Fig. 3A, the proportion of cells in early apoptotic stage (Annexin $\mathrm{V}^{+} / 7-\mathrm{AAD}^{-}$) was increased from 2.70 to $18.00 \%$ and those in the late apoptotic stage (Annexin $\mathrm{V}^{+} / 7-\mathrm{AAD}^{+}$) were also increased from 17.36 to $39.72 \%$. Statistical analysis indicated that knockdown of STIM1 significantly promotes cell early- and late-stage apoptosis in SW1116 cells $(\mathrm{P}<0.001$; Fig. 3B) which provides evidence that STIM1 silencing may induce apoptosis in SW1116 cells.

To further uncover the molecular mechanism underlying cell apoptosis promoted by STIM1 silencing, the levels of Bad and Bax expression, and PARP cleavage were examined to detect apoptosis following silencing of STIM1 in SW1116 cells. The qPCR analysis indicated that expression levels of Bad and Bax were increased in SW1116 cells following STIM1 silencing ( $\mathrm{P}<0.01$ and $\mathrm{P}<0.001$; Fig. $3 \mathrm{C})$. Furthermore, an increase in PARP cleavage was observed in SW1116 cells with silenced STIM1, indicating a higher proportion of apoptotic cells as a consequence of STIM1 silencing (Fig. 3D).

\section{Discussion}

Despite the ongoing advancement of our understanding of cancer progression and metastasis in the last decade, a number of oncogenes remain poorly understood. Previous studies indicate that $\mathrm{Ca}^{2+}$ channels are critical regulators in cancer $(20,21)$. 

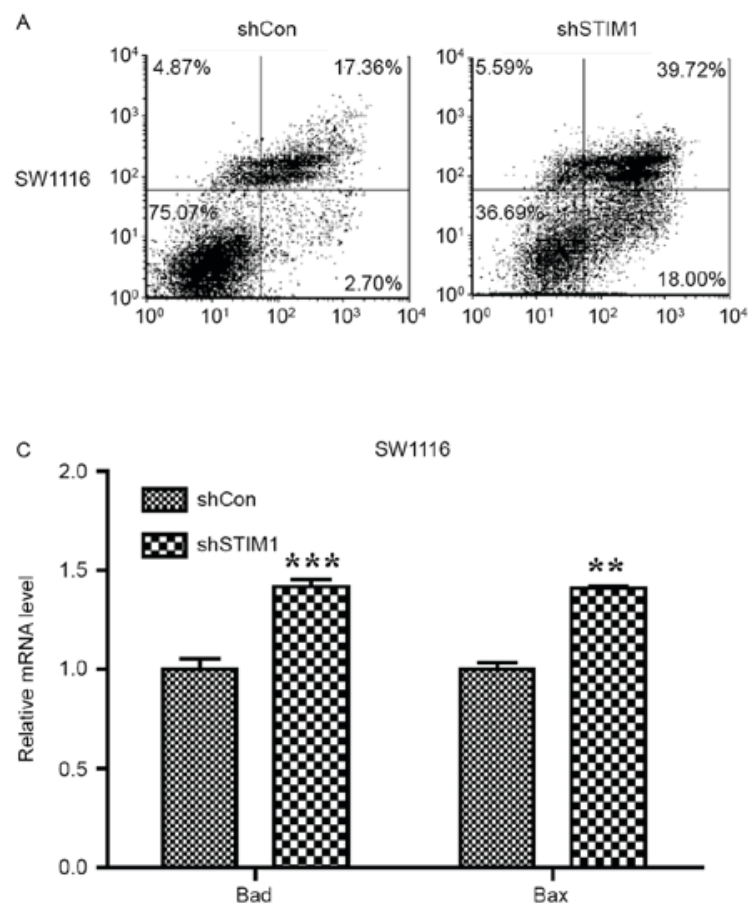
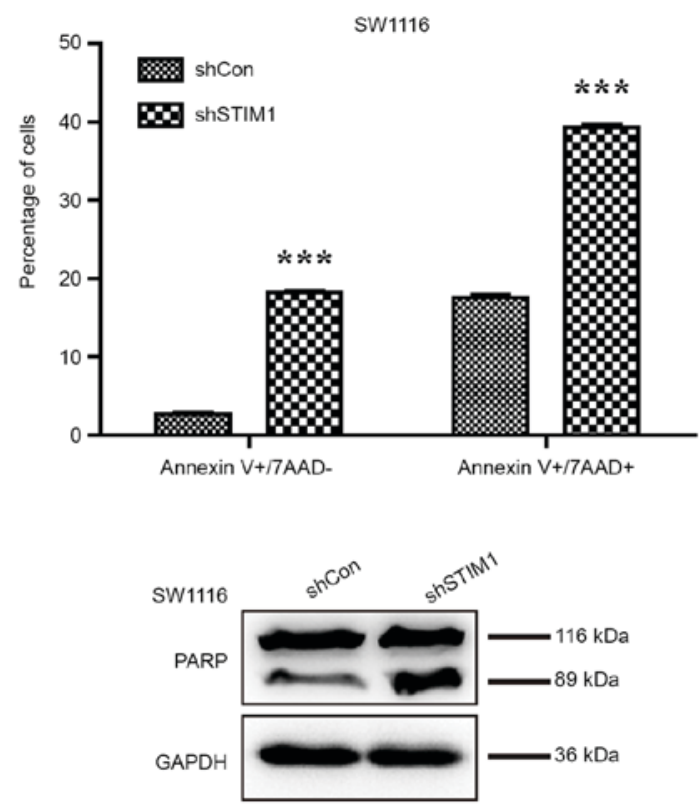

Figure 3. Knockdown of STIM1 induces cell apoptosis via activation of the mitochondrial signalling pathway. (A) Representative images of cell apoptosis of SW1116 cells following shSTIM1 infection analyzed using flow cytometry and Annexin V/7-AAD double staining. (B) Quantification of the proportions of SW1116 cells corresponding to early (Annexin $\mathrm{V}^{+} / 7-\mathrm{AAD}^{-}$) and late (Annexin $\mathrm{V}^{+} / 7-\mathrm{AAD}^{+}$) apoptotic cells. Upregulation of (C) Bad and Bax, and (D) cleaved PARP measured by western blot analysis. The experiment was performed in triplicate and repeated in triplicate. ${ }^{* *} \mathrm{P}<0.01,{ }^{* * *} \mathrm{P}<0.001 \mathrm{vs}$. shCon. $\mathrm{STIM} 1$, stromal interaction molecule 1; shSTIM1, shRNA targeting human STIM1; shCon, non-silencing shRNA; CRC, colorectal cancer; PARP, poly(ADP-ribose) polymerase; BAD, B-cell lymphoma-2 associated death promoter; Bax, B-cell lymphoma-2 associated X protein; 7-AAD, 7-aminoactinomycin D.

As a $\mathrm{Ca}^{2+}$ sensor, STIM1 is required for a number of important physiological and pathological processes. Previous studies indicate that STIM1 may be a potential therapeutic target in numerous types of cancer $(10,11,17)$. In breast cancer, overexpression of STIM1 is able to promote metastasis and invasion of cancer cells (15). In lung cancer, STIM1 is upregulated in the carcinoma relative to the para-carcinoma tissues (22). Additionally, STIM1 overexpression promotes CRC cell migration by inducing cyclo-oxygenase 2 expression and is associated with CRC progression (17); however, the role of STIM1 in CRC cell proliferation has not been fully elucidated.

Results of the present study suggest that knockdown of STIM1 inhibits the proliferation and colony formation ability of CRC cells, which is consistent with results from a previous study (23). Furthermore, Annexin V/7-AAD double staining confirmed that knockdown of STIM1 increased the proportion of early and late apoptotic cells. Cleavage of PARP is one of the most commonly used diagnostic tools for the detection of apoptosis in numerous cell types (24). In the present study, the expression of PARP cleavage was increased in STIM1-silenced cells. Furthermore, knockdown of STIM1 increased the expression of the pro-apoptotic mitochondrial genes Bax and Bad in SW1116 cells. Therefore, it may be inferred that the cell proliferation inhibition by STIM1 silencing in CRC was possibly due to the induction of mitochondria-associated apoptosis.

In conclusion, the results of the present study demonstrate that STIM1 knockdown by lentivirus-mediated shRNA may inhibit the proliferation of CRC cells, which may be a result of induced apoptosis. These results also for the first time highlight the crucial role of STIM1 in CRC progression. Further study is required to illuminate the potential value of STIM1-targeted therapy in vivo.

\section{Acknowledgements}

Not applicable.

\section{Funding}

The present study was supported by the Natural Science Foundation of Ningbo (grant nos. 2013A610224 and 2016A610135), the Municipal Key Disciplines of Ningbo (grant no. 2013-88), the Medical Foundation of Ningbo (grant no. 2011B10), the Social Development and Scientific and Technological Projects Foundation of Ningbo (grant no. 2014C50068) and the Huamei Foundation of Ningbo No. 2 Hospital (grant nos. 2015HMKJ08 and 2016HMKJ26).

\section{Availability of data and materials}

The datasets used and/or analyzed during the current study are available from the corresponding author on reasonable request.

\section{Authors' contributions}

DY performed the vector construction experiments and drafted the manuscript. XD and KL participated in the research design, reviewed the literature and examined the 
data. YX and JZ participated in the qPCR and western blot experiments. MD and HY participated in the cellular function experiments. ZK participated in the data analysis and figure format.

\section{Ethics approval and consent to participate}

Not applicable.

\section{Consent for publication}

Not applicable.

\section{Competing interests}

The authors declare that they have no competing interests.

\section{References}

1. Torre LA, Bray F, Siegel RL, Ferlay J, Lortet-Tieulent J and Jemal A: Global cancer statistics, 2012. CA Cancer J Clin 65: 87-108, 2015

2. Siegel R, Desantis C and Jemal A: Colorectal cancer statistics, 2014. CA Cancer J Clin 64: 104-117, 2014.

3. Fearon ER: Molecular genetics of colorectal cancer. Ann N Y Acad Sci 768: 101-110, 1995.

4. Song M, Garrett WS and Chan AT: Nutrients, foods, and colorectal cancer prevention. Gastroenterology 148: 1244-1260, 2015.

5. Parker NJ, Begley CG, Smith PJ and Fox RM: Molecular cloning of a novel human gene (D11S4896E) at chromosomal region 11p15.5. Genomics 37: 253-256, 1996.

6. Prevarskaya N, Skryma R and Shuba Y: Calcium in tumour metastasis: New roles for known actors. Nature Rev Cancer 11: 609-618, 2011

7. Roos J, DiGregorio PJ, Yeromin AV, Ohlsen K, Lioudyno M, Zhang S, Safrina O, Kozak JA, Wagner SL, Cahalan MD, et al: STIM1, an essential and conserved component of store-operated Ca2+ channel function. J Cell Biol 169: 435-445, 2005

8. Hogan PG, Lewis RS and Rao A: Molecular basis of calcium signaling in lymphocytes: STIM and ORAI. Annu Rev Immunol 28: 491-533, 2010.

9. Hu J, Qin K, Zhang Y, Gong J, Li N, Lv D, Xiang R and Tan X: Downregulation of transcription factor Oct4 induces an epithelial-to-mesenchymal transition via enhancement of $\mathrm{Ca} 2+$ influx in breast cancer cells. Biochem Biophys Res Commun 411: 786-791, 2011

10. Hou MF, Kuo HC, Li JH, Wang YS, Chang CC, Chen KC, Chen WC, Chiu CC, Yang S and Chang WC: Orai1/CRACM1 overexpression suppresses cell proliferation via attenuation of the store-operated calcium influx-mediated signalling pathway in A549 lung cancer cells. Biochim Biophys Acta 1810: 1278-1284, 2011.

11. Chen YF, Chiu WT, Chen YT, Lin PY, Huang HJ, Chou CY, Chang HC, Tang MJ and Shen MR: Calcium store sensor stromal-interaction molecule 1-dependent signaling plays an important role in cervical cancer growth, migration, and angiogenesis. Proc Natl Acad Sci USA 108: 15225-15230, 2011.
12. Fedida-Metula S, Feldman B, Koshelev V, Levin-Gromiko U, Voronov E and Fishman D: Lipid rafts couple store-operated Ca2+ entry to constitutive activation of PKB/Akt in a Ca2+/calmodulin-, Src- and PP2A-mediated pathway and promote melanoma tumor growth. Carcinogenesis 33: 740-750, 2012.

13. van de Vijver MJ, He YD, van't Veer LJ, Dai H, Hart AA, Voskuil DW, Schreiber GJ, Peterse JL, Roberts C, Marton MJ, et al: A gene-expression signature as a predictor of survival in breast cancer. N Engl J Med 347: 1999-2009, 2002.

14. Faehling M, Kroll J, Föhr KJ, Fellbrich G, Mayr U, Trischler G and Waltenberger J: Essential role of calcium in vascular endothelial growth factor A-induced signaling: Mechanism of the antiangiogenic effect of carboxyamidotriazole. FASEB J 16 1805-1807, 2002

15. Yang S, Zhang JJ and Huang XY: Orai1 and STIM1 are critical for breast tumor cell migration and metastasis. Cancer cell 15: 124-134, 2009.

16. Flourakis M, Lehen'kyi V, Beck B, Raphaël M, Vandenberghe M, Abeele FV, Roudbaraki M, Lepage G, Mauroy B, Romanin C, et al: Orail contributes to the establishment of an apoptosis-resistant phenotype in prostate cancer cells. Cell Death Dis 1: e75, 2010

17. Wang JY, Sun J, Huang MY, Wang YS, Hou MF, Sun Y, He H, Krishna N, Chiu SJ, Lin S et al: STIM1 overexpression promotes colorectal cancer progression, cell motility and COX-2 expression. Oncogene 34: 4358-4367, 2015

18. Zhang Z,Liu X,Feng B,LiuN,Wu Q,Han Y,Nie Y,Wu K, Shi Y and Fan D: STIM1, a direct target of microRNA-185, promotes tumor metastasis and is associated with poor prognosis in colorectal cancer. Oncogene 35: 6043, 2016.

19. Livak KJ and Schmittgen TD: Analysis of relative gene expression data using real-time quantitative PCR and the 2(-Delta Delta C(T)) method. Methods 25: 402-408, 2001.

20. Monteith GR, Davis FM and Roberts-Thomson SJ: Calcium channels and pumps in cancer: Changes and consequences. J Biol Chem 287: 31666-31673, 2012.

21. Chen YF, Chen YT, Chiu WT and Shen MR: Remodeling of calcium signaling in tumor progression. J Biomed Sci 20: 23, 2013.

22. Li W, Zhang M, Xu L, Lin D, Cai S and Zou F: The apoptosis of non-small cell lung cancer induced by cisplatin through modulation of STIM1. Exp Toxicol Pathol 65: 1073-1081, 2013.

23. Li G, Zhang Z, Wang R, Ma W, Yang Y, Wei J and Wei Y: Suppression of STIM1 inhibits human glioblastoma cell proliferation and induces G0/G1 phase arrest. J Exp Clin Cancer Res 32: 20, 2013.

24. Bressenot A, Marchal S, Bezdetnaya L, Garrier J, Guillemin F and Plénat F: Assessment of apoptosis by immunohistochemistry to active caspase- 3 , active caspase- 7 , or cleaved PARP in monolayer cells and spheroid and subcutaneous xenografts of human carcinoma. J Histochem Cytochem 57: 289-300, 2009

(i) $(-)$ This work is licensed under a Creative Commons Attribution-NonCommercial-NoDerivatives 4.0 International (CC BY-NC-ND 4.0) License. 